Ingeniería

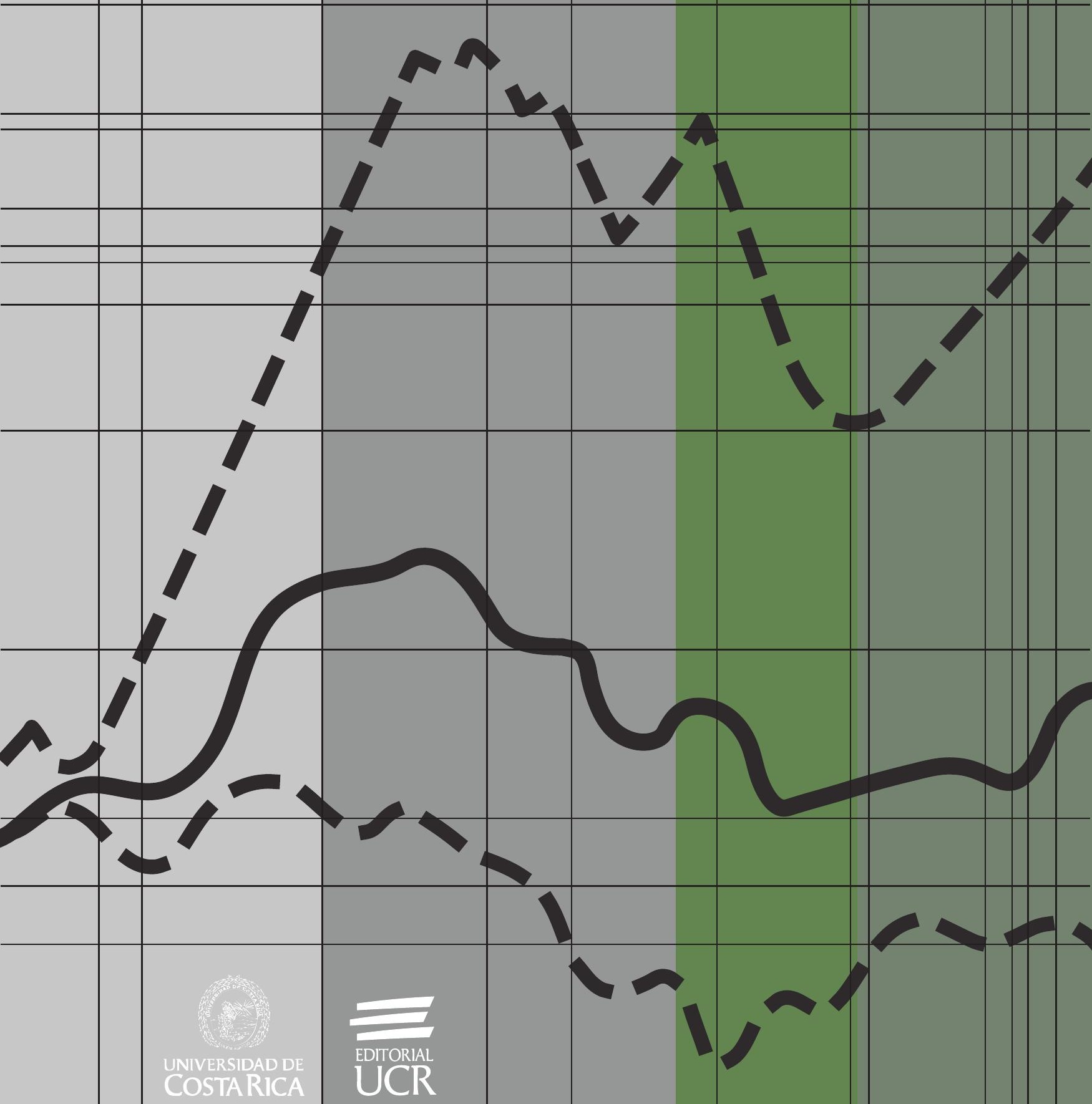




\title{
Comportamiento experimental a flexión de losas de puentes de concreto, reforzadas externamente con CFRP
}

\section{Experimental Flexural Behavior of Concrete Bridge Slabs, Externally Reinforced with CFRP}

\author{
Ing. Alejandro Navas Carro, M.Sc \\ Universidad de Costa Rica, Laboratorio Nacional de Materiales, Costa Rica \\ alejandro.navas@ucr.ac.cr \\ Ing. Carolina Fonseca Mojica \\ Universidad de Costa Rica, Costa Rica \\ carofons_93@hotmail.com
}

Recibido: 14 de abril 2018

Aceptado: 23 de abril 2018

\section{Resumen}

Se evaluó el comportamiento a flexión de secciones de losas de puentes de concreto que fueron sometidas a un estado de agrietamiento y posteriormente reparadas con polímeros reforzados con fibras de carbono (CFRP) para estudiar la posibilidad de utilizar este tipo de refuerzo en losas de puentes existentes en Costa Rica.

Se construyeron tres losas de concreto: un espécimen de tipo control (sin refuerzo con CFRP) y dos especímenes para evaluar el refuerzo con CFRP. El esquema de carga consistió en colocar las losas sobre tres apoyos simples y aplicar dos cargas puntuales que generasen momento positivo y negativo. Se sometieron las losas por reforzar a un $80 \%$ de su carga última para generar agrietamiento que requiriese reparación. Se repararon las grietas cuyos anchos de grieta superaron los $0.3 \mathrm{~mm}$ mediante inyección de epóxico y se reforzaron los dos especímenes con CFRP en las zonas de momento positivo y negativo. Finalmente se cargaron los tres especímenes hasta la falla.

Se obtuvo que las losas reforzadas con CFRP fueron $36 \%$ más resistentes que la losa control, lo que sugiere que este tipo de reforzamiento puede ser utilizado en losas de puentes existentes en el país. Además, se compararon los resultados experimentales de este proyecto con los resultados de la investigación de Carranza (2017) y se concluyó que ambas investigaciones dieron resultados similares, con porcentajes de diferencia entre un $10 \%$ y $14 \%$.

\section{Palabras clave}

Refuerzo a flexión, precarga, agrietamiento, reparación, resistencia. 


\begin{abstract}
The flexural behavior of bridge concrete slab sections that were subjected to a level of cracking and were later repaired with carbon fiber reinforced polymers (CFRP) was evaluated in order to study the possibility of using this type of strengthening in existent bridge slabs in Costa Rica.

Three concrete slabs were built: the first was a control specimen (which was not strengthened with CFRP) and the two others were meant to evaluate the effect of CFRP strengthening. The loading scheme consisted in placing the slabs on three simple supports and applying two concentrated loads, in order to generate positive and negative moment regions. The slabs meant for strengthening were initially loaded with an $80 \%$ of their ultimate strength with the purpose of generating cracks that needed repairing. The cracks whose crack widths exceeded $0.3 \mathrm{~mm}$ were repaired by epoxy injection and then the two slabs were strengthened with CFRP in the positive and negative moment regions. Finally the three specimens were loaded until failure.

The slabs reinforced with CFRP were 36\% stronger than the control slab, which suggests that this type of strengthening can be used in existing bridge slabs in the country. Additionally, the experimental results obtained in this project were compared with the experimental results from the project of Carranza (2017) and both projects had similar results, with difference percentages varying between $10 \%$ and $14 \%$.
\end{abstract}

Keywords

Flexural reinforcement, loading, cracking, repairing, resistance. 


\section{INTRODUCCIÓN}

Actualmente, en Costa Rica existen puentes que fueron construidos entre la década de 1940 y 1970. Estos puentes fueron diseñados con códigos de diseño antiguos y para un flujo vehicular menor que el presente. Con el paso del tiempo y su operación, las losas de concreto sufren un proceso de agrietamiento que reduce gradualmente su inercia y por ende su resistencia a flexión. Sumado a estas condiciones, se encuentra el escaso o nulo mantenimiento que se le brinda a estas estructuras. Los puentes suelen estar ubicados en vías que no poseen una adecuada redundancia, además estas estructuras son costosas y necesarias para el transporte de bienes y servicios. Por este motivo se considera importante la investigación de algún método de refuerzo de losas que se pueda implementar con una mínima interrupción al tránsito y que permita aumentar su resistencia para adecuarlas a las cargas vehiculares actuales, reparar daños y alargar su vida útil.

El sistema de polímeros reforzados con fibras de carbono (CFRP, por sus siglas en inglés) consiste en fibras finas compuestas de cristales de grafito embebidas en una matriz de resina, cuya función es mantener las fibras unidas, protegerlas y trasmitir la carga a estas fibras (Uomoto, Mutsuyoshi, Katsuki y Misra, 2002). En el caso del refuerzo con CFRP, la resina puede ser aplicada in situ sobre la fibra. Entre las ventajas de este tipo de refuerzo se encuentran su alta resistencia, bajo peso y resistencia a la corrosión. Esto permite utilizar este material en estructuras de concreto con una mínima afectación a las cargas muertas de la estructura. Entre sus principales desventajas se incluye que este material falla en forma frágil a deformaciones muy bajas, por lo que resulta inadecuado utilizar este sistema en elementos donde sea necesario un comportamiento dúctil.

A nivel nacional se han realizado investigaciones acerca del comportamiento de vigas de concreto reforzado con fibras de carbono. En cuanto a investigaciones acerca del comportamiento a flexión de vigas reforzadas con CFRP, se han desarrollado dos proyectos de investigación en la Universidad de Costa Rica. En el proyecto de graduación "Evaluación del comportamiento de vigas de concreto armado reforzadas externamente con fibras de carbono" de Osejo (2012) se construyeron cuatro vigas de concreto reforzado con acero y posteriormente se reforzaron externamente con fibras de carbono. Posteriormente se fallaron en condición simplemente apoyada y se compararon los resultados de resistencia a la flexión de las vigas reforzadas con CFRP con la resistencia a la flexión de dos vigas control que no fueron reforzadas externamente con CFRP. Cabe destacar que en los resultados se obtuvo un $65 \%$ de aumento en la resistencia de las vigas que fueron reforzadas externamente con CFRP con respecto a las vigas control.

El proyecto de investigación "Evaluación del comportamiento a flexión en vigas de concreto reforzadas con fibras de carbono, una vez que han sido sometidas a un estado de carga" de Mata (2014) le dio continuidad a la investigación elaborada por Osejo (2012). En este proyecto se construyeron el mismo número de vigas con las mismas dimensiones y características de refuerzo y resistencia de materiales. La diferencia radica en que se sometieron las vigas a un estado de precarga equivalente a un $80 \%$ de su capacidad. Posteriormente, se repararon y reforzaron cuatro vigas mediante el sistema CFRP. Finalmente, se llevó a falla la totalidad de las muestras y se realizó una comparación entre la resistencia de los especímenes reparados con CFRP y dos 
vigas control sin refuerzo; asimismo, se compararon los resultados con los resultados obtenidos por Osejo (2012). En este caso, el autor obtuvo un porcentaje de aumento de un $59 \%$ en la resistencia de las vigas que fueron reforzadas con CFRP, con respecto a las vigas control.

Con respecto al comportamiento en cortante de vigas reforzadas con fibras de carbono, se han realizado cinco proyectos de graduación en la Universidad de Costa Rica. En estos se ha evaluado el comportamiento de vigas reforzadas perimetralmente con CFRP y reforzadas con configuración U.

Recientemente Carranza (2017) realizó la primera investigación a nivel nacional acerca del comportamiento de losas de puentes reforzadas externamente en flexión con fibra de carbono. Este trabajo consistió en la construcción de tres losas de concreto reforzado con acero, donde buscó construir losas similares a las típicas de puentes que se encuentran en Costa Rica. Dos losas fueron reforzadas externamente con el sistema CFRP y posteriormente fueron falladas, los resultados se compararon con una losa control que no fue reforzada. Los resultados de la investigación de Carranza (2017) fueron comparados con los resultados obtenidos en esta investigación con el propósito de realizar comparaciones y darle continuidad a este tema.

En cuanto a investigaciones a nivel internacional, se ha realizado una gran cantidad de pruebas en elementos de concreto reforzado. Se destaca el trabajo de Arduini, Nanni y Romagnolo (2004), quienes determinaron el desempeño de losas de concreto reforzado en una dirección, reforzadas externamente con CFRP. En esta investigación se construyeron 26 losas a escala natural que fueron falladas en condición de apoyo simple, con diferentes configuraciones que permitieron evaluar zonas de momento positivo y negativo, y con diferentes cantidades de acero de refuerzo. Como resultado, se encontró que las losas reforzadas con CFRP son capaces de aumentar su capacidad significativamente, en algunos casos hasta en un $122 \%$.

Así, se trabajó según el siguiente esquema:

\subsection{Objetivos}

\section{Objetivo General}

Estudiar el comportamiento a flexión de secciones de losas de concreto que han sido sometidas a un estado de agrietamiento y posteriormente han sido reparadas con fibras de carbono.

\section{Objetivos Específicos}

- Construir dos tipos de losas, de igual geometría y refuerzo, que sean similares a losas de puentes típicas en Costa Rica, donde un diseño contemple el uso de fibras de carbono y el otro diseño constituya una losa de control.

- Generar un estado de agrietamiento en dos losas que justifique su reparación y reforzamiento con fibra de carbono. 
- Evaluar la resistencia a flexión de las losas que fueron reparadas con fibras de carbono y comparar estos resultados con la losa control.

- Estudiar el comportamiento de los especímenes y su tipo de falla al ser sometidos a su carga última.

- Comparar los resultados obtenidos en este proyecto con los resultados obtenidos en el trabajo de Carranza (2017).

\section{METODOLOGÍA}

La metodología utilizada para la elaboración del proyecto se muestra en la figura 1. El desarrollo del proyecto contó con tres etapas principales: a) investigación bibliográfica y diseño de especímenes, b) proceso experimental y c) análisis de resultados.

En la primera etapa se realizó un estudio bibliográfico de la información existente acerca del tema de estudio, principalmente las propiedades del sistema de refuerzo externo con CFRP, estudios previos y normativa de diseño. Este conocimiento fue utilizado para determinar en forma teórica la capacidad de las losas y realizar comparaciones con los resultados experimentales.

La segunda etapa contempló todo el proceso experimental del proyecto. En primer lugar se construyeron los tres especímenes, posteriormente se aplicó un $80 \%$ de la carga última de las losas, lo que generó daños por agrietamiento y se falló la losa control. Se repararon y reforzaron con CFRP dos de las losas y la losa que no se reparó fue considerada como losa control con el fin de realizar comparaciones con las otras. Finalmente, se cargaron las losas reforzadas con CFRP hasta la falla. Adicionalmente se hicieron pruebas de resistencia al concreto y acero de refuerzo con el propósito de calcular con mayor exactitud el comportamiento y resistencia de las losas.

La última etapa consistió en el análisis de los resultados obtenidos en forma experimental y teórica. En esta fase se realizaron comparaciones entre los resultados de las losas que fueron reforzadas externamente con el sistema de CFRP y la losa control, y comparaciones entre los resultados de las losas reforzadas con el sistema CFRP y los resultados obtenidos en el proyecto de Carranza (2017).

\section{PROPIEDADES DE LAS LOSAS Y CONFIGURACIÓN DE LOS ENSAYOS}

Como fue mencionado previamente, el proyecto buscó analizar el comportamiento a flexión de losas con características similares a losas de puentes construidas en el país. Las característica s geométricas, las propiedades de los materiales, la configuración del ensayo, y el tipo y ubicación del refuerzo replican el proyecto de Carranza (2017), pues se busca realizar comparaciones entre los resultados obtenidos en ambas investigaciones.

Se seleccionó una losa experimental de $5.3 \mathrm{~m}$ de largo, $20 \mathrm{~cm}$ de espesor y $50 \mathrm{~cm}$ de ancho. Como acero de refuerzo, se seleccionó varilla \#5, grado 60 que cumpliese con la norma ASTM A615 (American Standard of Testing and Materials, 2016). Se eligió 
una resistencia del concreto de $280 \mathrm{~kg} / \mathrm{cm} 2$. Se utilizó una separación de $40 \mathrm{~cm}$ para el acero principal de la losa y una separación de $20 \mathrm{~cm}$ para el acero secundario, tanto para la zona superior e inferior de la losa. Las características descritas previamente se ilustran en la figura 2 .

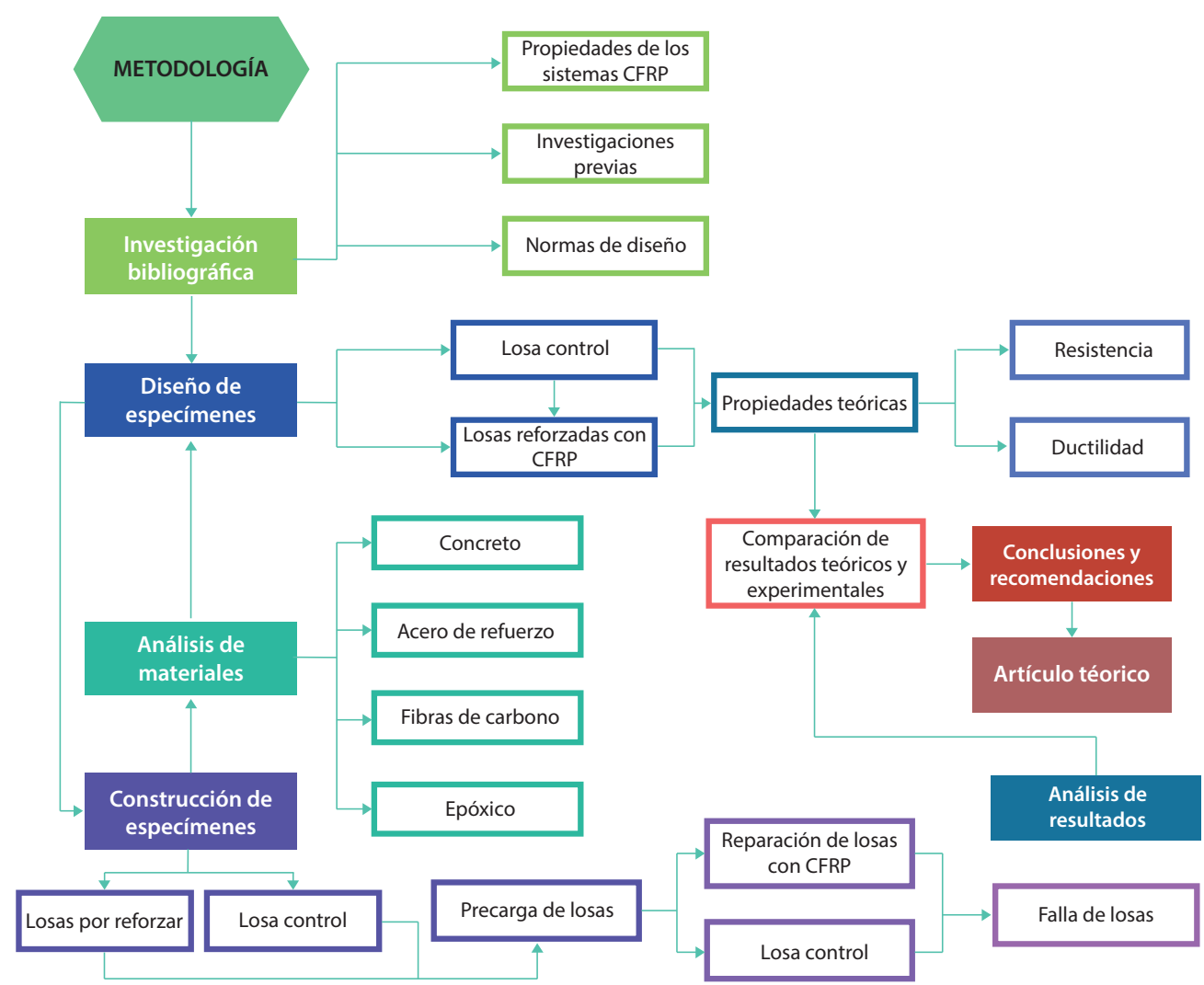

Figura 1. Metodología para la elaboración del proyecto de graduación

La configuración del ensayo buscó modelar una losa apoyada en tres vigas, que es cargada por el paso de un camión de diseño. Ambas llantas del camión se representaron con dos cargas puntuales separadas a 1.8 m entre sí. La ubicación de estas cargas estuvo sujeta a generar el máximo momento en la losa, el cual corresponde a momento negativo en la zona central de la losa y se da cuando el camión se ubica centrado en la losa, con cada llanta a $0.9 \mathrm{~m}$ del centro.

La configuración de carga y apoyos seleccionados para las pruebas generaron un máximo momento (negativo) en el centro de la losa y momentos positivos en los claros externos de la losa. Estas zonas son las que sufren el mayor daño (agrietamiento) y definen la capacidad de la losa, por lo que corresponden a las zonas en donde se colocó el refuerzo externo de fibra de carbono. Cabe destacar que estas zonas fueron reforzadas con una única capa del sistema de CFRP, tanto en los claros de las losas donde se genera el momento positivo, como en la zona central donde se genera el momento negativo. 
La figura 3 muestra la configuración de la prueba, los puntos de carga y las zonas por reforzar con el sistema CFRP.

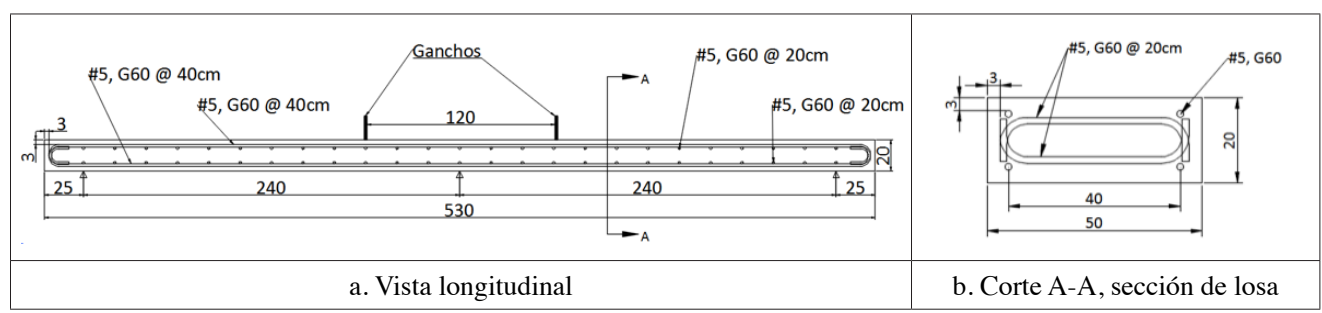

Figura 2. Vistas de la losa, cotas en centímetros

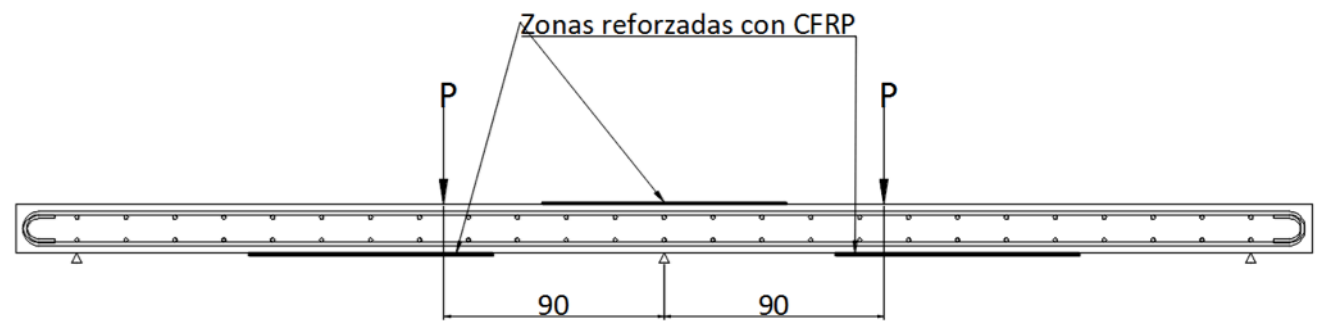

Figura 3. Ubicación de las cargas y de las zonas por reforzar con CFRP

\subsection{Instrumentación y esquema de carga}

Las losas por precargar fueron instrumentadas con dos LVDTs, cada uno ubicado bajo los puntos de carga en ambos claros, pues aquí se generarían las máximas deflexiones.

Sobre las losas se colocó una viga de acero de $30 \mathrm{~cm}$ de peralte, apoyada sobre dos rodillos ubicados justo en los puntos de carga: a $0.9 \mathrm{~m}$ del centro de las losas. Se utilizaron marcos de carga con gatos hidráulicos para cargar sobre esta viga de carga, la cual a su vez transmitió las cargas a las losas en forma de dos cargas puntuales a través de los rodillos (ver figura 4 ).

Para la precarga de las dos primeras losas, se aplicaron cinco ciclos de carga que alcanzaron un máximo de un $80 \%$ de la capacidad última de las losas. Se decidió seleccionar un $80 \%$ de la carga nominal de las losas pues fue un valor que generaría agrietamiento que requiriese ser reparado y a su vez no causaría la cedencia del acero de refuerzo. De acuerdo con estas consideraciones, en cada ciclo de carga se aplicaron $51 \mathrm{KN}$ en cada punto de carga y posteriormente se descargaron hasta $10 \mathrm{KN}$ en cada punto de carga.

Para la tercera losa se aplicó un único ciclo de carga en forma monotónica hasta el $80 \%$ de la capacidad de la losa y posteriormente se descargó el elemento. 


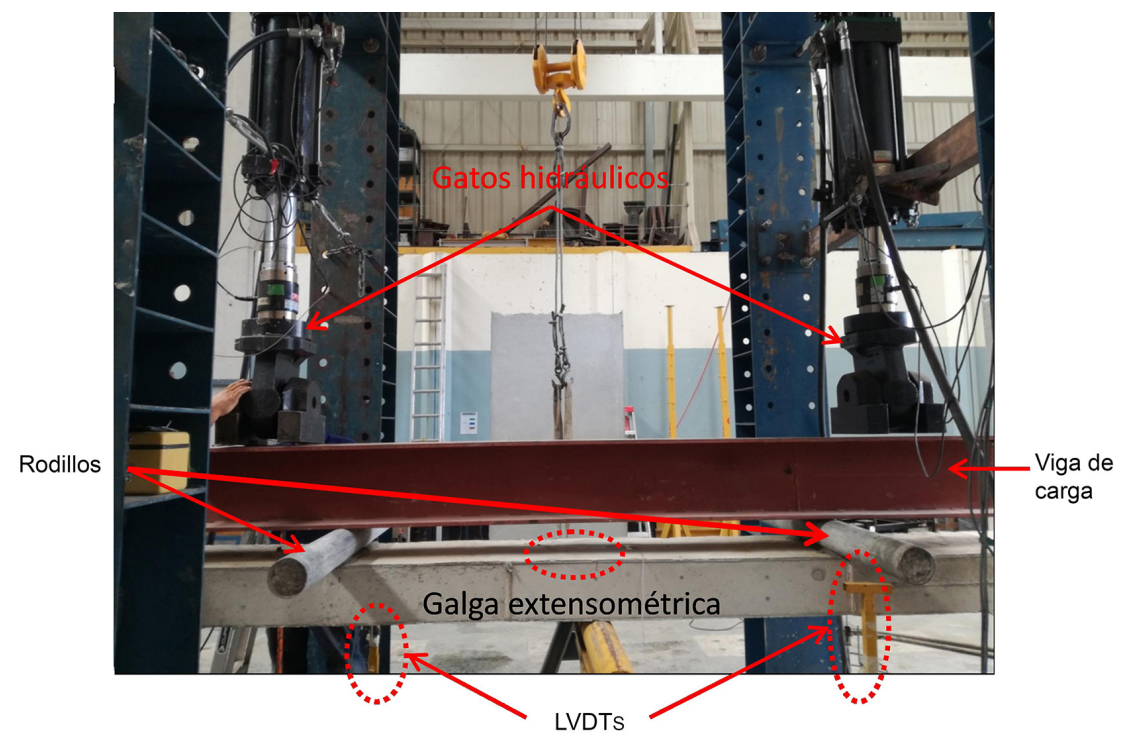

Figura 4. Esquema de precarga y carga de las losas

\section{MATERIALES}

A continuación se presentan las propiedades de los materiales utilizados en la construcción de las losas. Con estos datos se realizaron los cálculos de las resistencias teóricas de las losas control y reforzadas con el sistema CFRP, así como deflexiones, entre otros.

\subsection{Concreto}

Tabla 1. Resistencia de los cilindros de concreto a diferentes edades

\begin{tabular}{cccc}
\hline Edad de falla & Área $\left(\mathrm{mm}^{2}\right)$ & Carga axial $(\mathrm{KN})$ & Resistencia $(\mathrm{MPa})$ \\
\hline 7 días & 8028.86 & 177.55 & 22.1 \\
28 días & 7965.57 & 257.90 & 32.4 \\
107 días & 7975.67 & 213.17 & 26.7 \\
\hline
\end{tabular}

\subsection{Acero de refuerzo}

Tabla 2. Propiedades del acero de refuerzo

\begin{tabular}{ccccc}
\hline $\begin{array}{c}\text { Área } \\
\left(\mathrm{cm}^{2}\right)\end{array}$ & $\begin{array}{c}\text { Esfuerzo de fluen- } \\
\text { cia } \mathrm{F}_{\mathrm{y}}(\mathrm{MPa})\end{array}$ & $\begin{array}{c}\text { Esfuerzo último } \\
\mathrm{F}_{\mathrm{u}}(\mathrm{MPa})\end{array}$ & $\begin{array}{c}\text { Módulo de } \\
\text { elasticidad } \mathrm{E}(\mathrm{MPa})\end{array}$ & $\begin{array}{c}\text { Elongación } \\
(\%)\end{array}$ \\
\hline 1.94 & 475.5 & 766.0 & $1.98 \times 106$ & 17.6 \\
\hline
\end{tabular}




\subsection{Sistema CFRP}

El sistema de refuerzo a base de polímeros de fibras de carbono (CFRP) se obtiene del conjunto tejido de fibra de carbono más la resina epóxica. Es importante señalar que para realizar diseños o análisis de elementos reforzados con este sistema, se deben utilizar las propiedades del conjunto CFRP, en lugar de las propiedades individuales de los componentes.

Tabla 3. Propiedades del sistema CFRP

\begin{tabular}{ll}
\hline Propiedad & \\
\hline Espesor de cada capa, $t_{f}$ & $0.508 \mathrm{~mm}$ \\
Módulo de elasticidad, $E_{f}$ & $5.17 \times 10^{4} \mathrm{MPa}$ \\
Resistencia última en tracción, $f_{f u}{ }^{*}$ & $662 \mathrm{MPa}$ \\
Deformación última en tracción, $\varepsilon_{f u}{ }^{*}$ & $0.0101 \mathrm{~mm} / \mathrm{mm}$ \\
\hline
\end{tabular}

Fuente: Sika, 2012

\section{RESULTADOS}

Para la presentación y análisis de resultados, se denominan las losas de la siguiente manera: la losa control como "LC", la losa reforzada 1 como "LR1" y la losa reforzada 2 como "LR2".

\subsection{Falla de losas}

\section{Losa control (LC)}

La figura 5 describe en forma gráfica el proceso de carga y falla de la losa control, pues presenta la carga total ejercida por el gato en función del desplazamiento de la cabeza del gato.

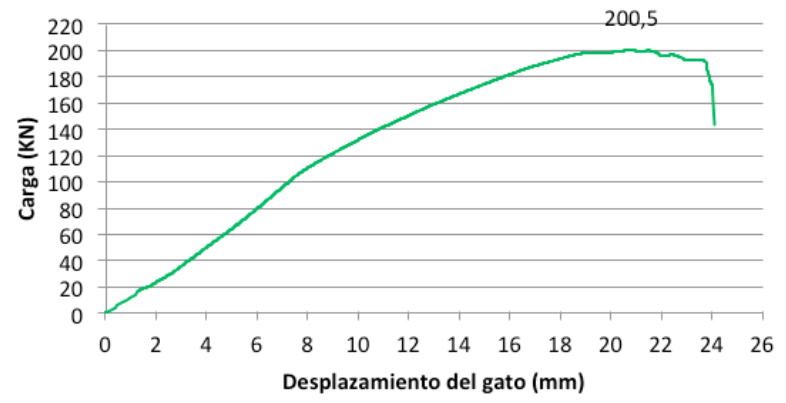

Figura 5. Carga total versus desplazamiento del gato hidráulico en la falla de la losa control 
La figura 5 ilustra que la losa control fue cargada monotónicamente hasta alcanzar la falla. Se ve una primera etapa en que se da deflexión conforme aumenta la carga; se muestran cambios de pendiente producto del agrietamiento del elemento y su consecuente pérdida de rigidez. Luego la carga y deformación continúan hasta alcanzar una carga de $198 \mathrm{KN}$, donde se presenta una caída de la pendiente en la gráfica que indica que se genera deformación sin incremento de la carga. La carga última soportada por el elemento fue de $200.5 \mathrm{KN}$.

Durante la falla de esta losa, se observó la formación de grietas verticales en ambas zonas de momento positivo, principalmente en la zona cerca de la aplicación de las cargas concentradas. Posteriormente, estas grietas se expandieron en forma inclinada, lo que indica que estas zonas estuvieron expuestas a esfuerzos de tracción y cortante (ver figura 6).

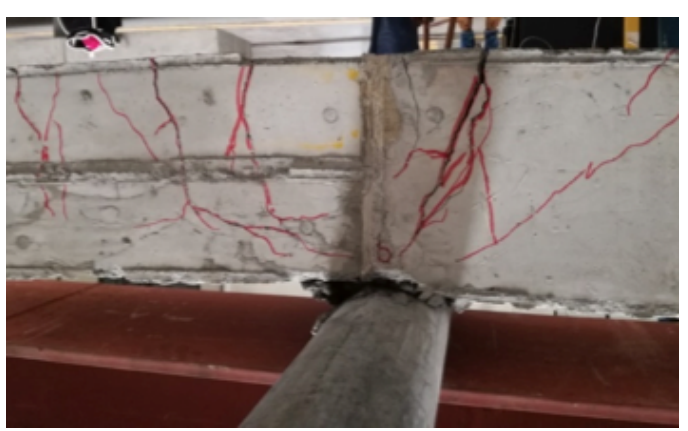

a. Claro derecho

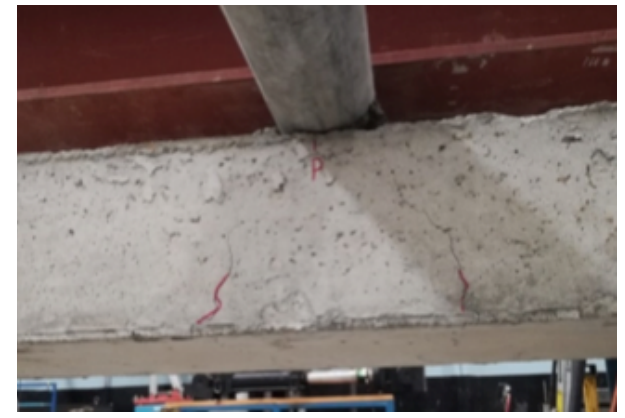

b. Claro izquierdo

Figura 6. Patrón de grietas en los claros de la losa control

La zona de momento negativo (máximo momento) presentó inicialmente grietas verticales (características de flexión) en la zona del apoyo. Al momento de la falla, se formó una grieta de cortante que inició en el apoyo y se extendió hasta la cara superior de la losa (ver figura 7).

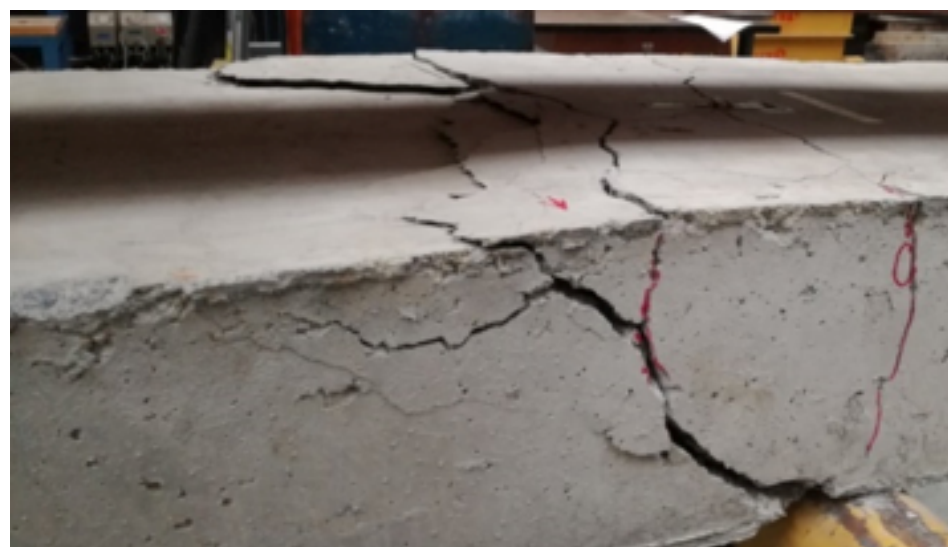

Figura 7. Patrón de grietas en la zona central de la losa, momento negativo 
La tabla 4 presenta la carga total que corresponde a la carga aplicada por el gato hidráulico, mientras que $\mathrm{P}_{\text {experimental }}$ representa cada carga concentrada, además se presentan el cortante y momento último soportado por la losa.

Tabla 4. Carga total soportada por la losa control

\begin{tabular}{cc}
\hline Carga total $(\mathrm{KN})$ & $\mathrm{P}_{\text {experimental }} *(\mathrm{KN})$ \\
200.5 & 100.3 \\
$\mathrm{M}_{\text {experimental }}(\mathrm{KN}-\mathrm{m})$ \\
47.5 \\
$\mathrm{~V}_{\text {experimental }}(\mathrm{KN})$ \\
85.3 \\
\hline *P
\end{tabular}

\subsection{Falla de losas reforzadas}

Losa reforzada 1 (LRI)

La figura 8 muestra el comportamiento del elemento durante la prueba, donde el LVDT 1 registró la deflexión en el claro izquierdo de la losa, bajo la carga puntual, y el LVDT 2 registró la deflexión en el claro derecho de la losa, bajo la carga puntual.

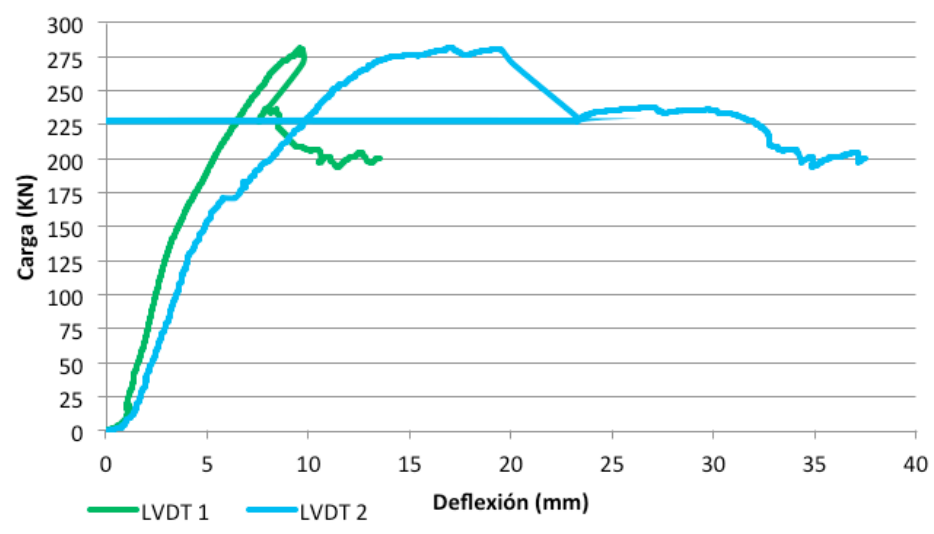

Figura 8. Carga versus deflexión en falla de losa LR1

$\mathrm{Al}$ analizar la figura 8 , se puede observar el comportamiento de la losa durante la prueba. El elemento se comportó en forma frágil pues se presentan incrementos de carga a muy bajas deformaciones y una pérdida de carga súbita asociada a su falla. En 
la primera zona de las gráficas (zona creciente) hubo ligeros cambios de pendiente a medida que aumentaba la carga debido a la pérdida de rigidez al agrietarse el elemento. Cuando se alcanza una carga de alrededor de $275 \mathrm{KN}$, se da una caída en la pendiente del elemento y una pequeña zona de comportamiento inelástico, que fue registrada principalmente por el LVDT2 en el claro derecho. La figura 9 muestra el claro derecho donde ocurrió la falla inicial, al propagarse grietas de cortante desde el punto de aplicación de la carga puntual hasta la zona inferior donde estaba la fibra y que causaron la delaminación de la fibra.

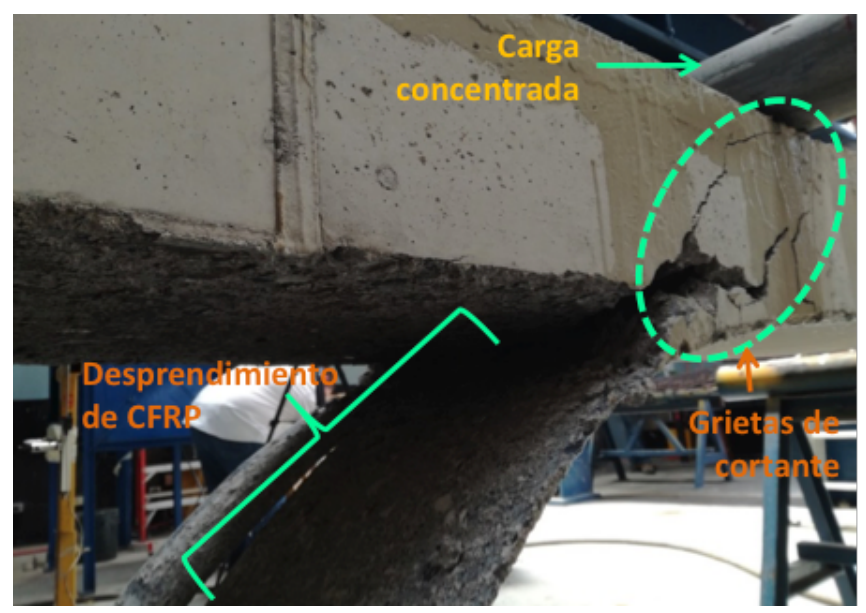

Figura 9. Grietas de cortante y desprendimiento de la fibra de carbono en el claro derecho de la losa LR1

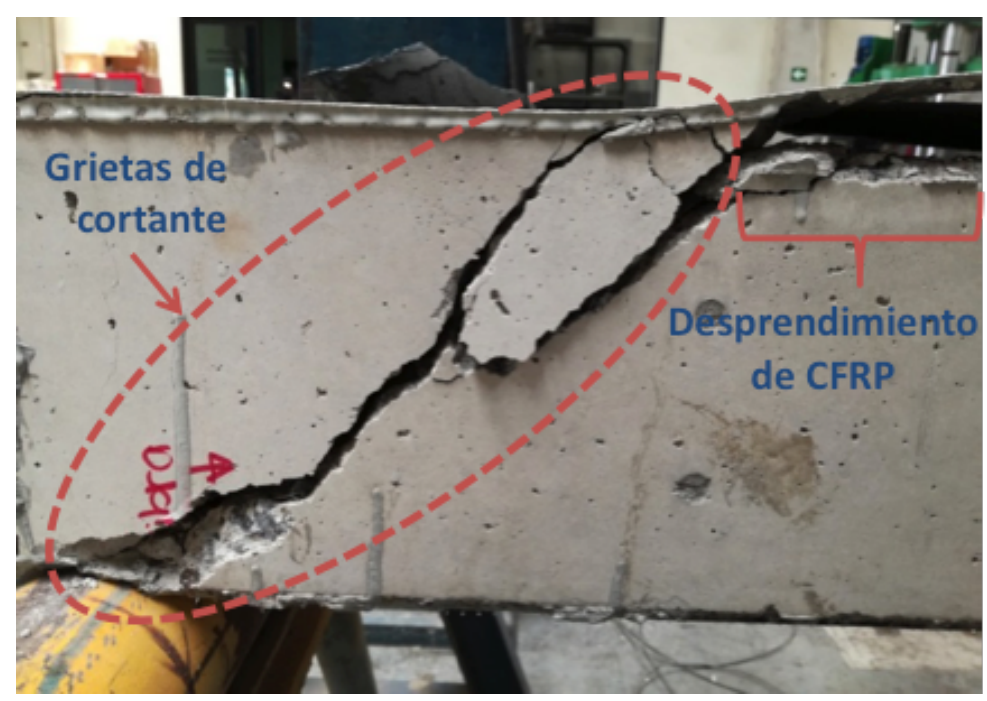

Figura 10. Grietas de cortante y desprendimiento de la fibra de carbono en el centro de la losa LR1 
Posterior a la pérdida de capacidad debido al desprendimiento de la fibra en el claro derecho, se continuó con el ensayo unos segundos más hasta que ocurrió otra falla por cortante sobre el apoyo central de la losa, la cual ocasionó que la fibra también se despegara (ver figura 10).

La tabla 5 presenta la carga soportada por la losa, el momento experimental y el cortante experimental.

Tabla 5. Carga total soportada por la losa LR1

\begin{tabular}{cc}
\hline $\begin{array}{c}\text { Carga total }(\mathrm{KN}) \\
281.6\end{array}$ & $\mathrm{P}_{\text {experimental }} *(\mathrm{KN})$ \\
& $\mathrm{M}_{\text {experimental }}(\mathrm{KN}-\mathrm{m})$ \\
& 66.0 \\
& $\mathrm{~V}_{\text {experimental }}(\mathrm{KN})$ \\
& 118.3 \\
\hline$* \mathrm{P}_{\text {experimental }}$ representa cada carga concentrada
\end{tabular}

Losa reforzada 2 (LR2)

De nuevo, se procedió a graficar los resultados adquiridos de la deflexión de los dos LVDTs y la carga aplicada (figura 11). El LVDT 1 registró la deflexión en el claro izquierdo de la losa, bajo la carga puntual, mientras que el LVDT 2 registró la deflexión en el claro derecho de la losa, bajo la carga puntual.

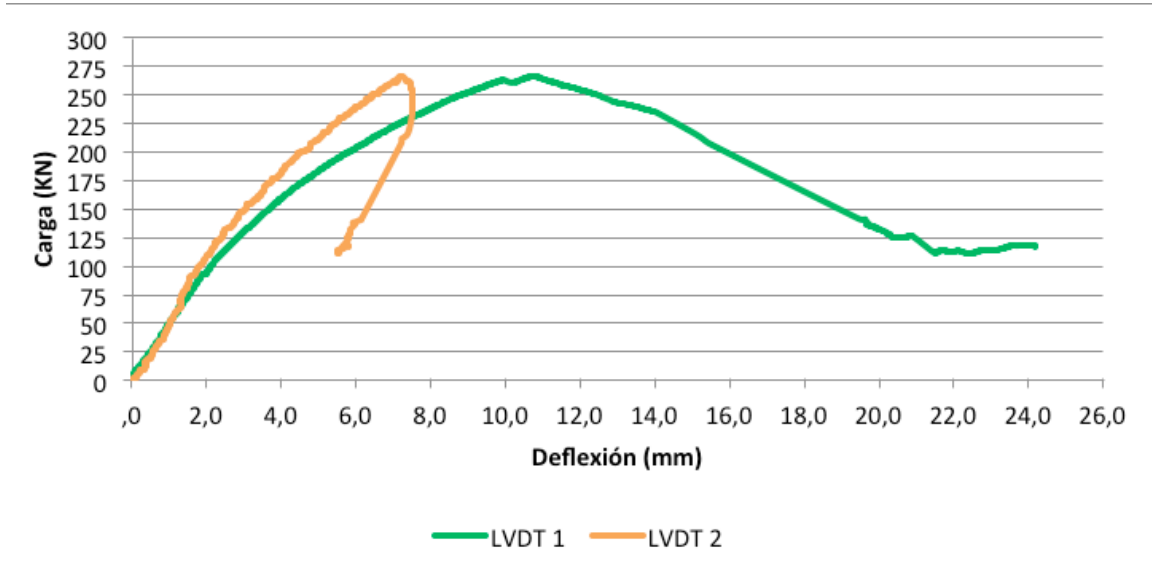

Figura 11. Carga versus deflexión en fallo de losa LR2

Al igual que en la losa LR1, la figura 11 muestra ligeros cambios de pendiente asociados a la pérdida de rigidez por agrietamiento. En este caso no se visualiza ninguna 
región de comportamiento inelástico cerca de la falla, la carga aumentó hasta un máximo de $266.2 \mathrm{KN}$ y la falla ocurrió en forma frágil en el centro de la losa, sobre el apoyo (ver figura 12). Esta inició con grietas de cortante en el apoyo que se extendieron hasta la superficie reforzada con fibra y ocasionaron su desprendimiento. En ese momento, se dio una caída de la capacidad de alrededor de un 58\% que marcó la falla de la losa. Al continuarse el ensayo durante unos segundos adicionales, la losa no presentó mayor capacidad, pues la carga no aumentó significativamente.

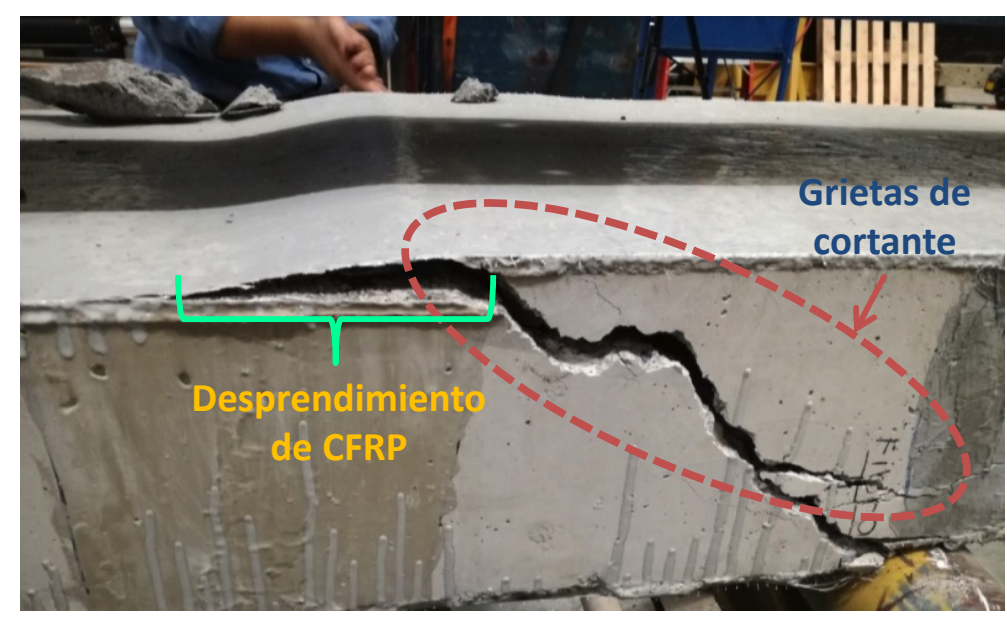

Figura 12. Grietas de cortante sobre el apoyo central y desprendimiento de la fibra de la losa LR2

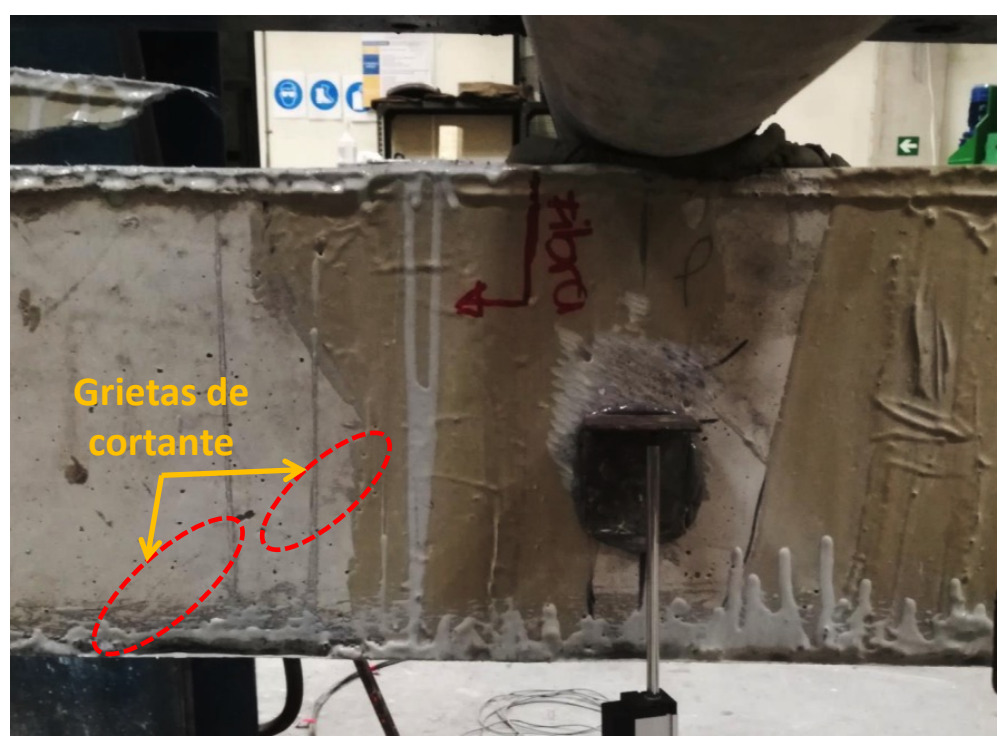

Figura 13. Agrietamiento en la zona bajo las cargas puntuales de la losa LR2 
La figura 13 presenta el agrietamiento en el claro izquierdo de la losa, cerca de los puntos de aplicación de carga. Se observan grietas de cortante pequeñas.

Se presenta en la tabla 6 la carga soportada por la losa, así el momento y cortante experimental.

Tabla 6. Carga total soportada por la losa LR2

\begin{tabular}{cc}
\hline Carga total $(\mathrm{KN})$ & $\mathrm{P}_{\text {experimental }} *(\mathrm{KN})$ \\
266.2 & 133.1 \\
$\mathrm{M}_{\text {experimental }}(\mathrm{KN}-\mathrm{m})$ \\
62.5 \\
$\mathrm{~V}_{\text {experimental }}(\mathrm{KN})$ \\
112.1 \\
\hline $\mathrm{P}_{\text {experimental }}$ representa cada carga concentrada
\end{tabular}

\subsection{Comparación con los resultados de Carranza (2017)}

Para finalizar, se realizó una comparación con los principales resultados obtenidos en la investigación de Carranza (2017).

En primer lugar, se compararon los resultados de la resistencia del concreto, el esfuerzo de fluencia y el módulo de elasticidad del acero de refuerzo (ver tabla 7).

Tabla 7. Resumen de resultados de propiedades de los materiales

\begin{tabular}{ccc}
\hline & Resultados Fonseca & Resultados Carranza (2017) \\
\hline$f_{c}^{\prime}$ concreto día de falla (MPa) & 26.7 & 44.7 \\
$f_{y}$ acero (MPa) & $475.7 \mathrm{a}$ & 520.6 \\
Módulo de elasticidad acero $(\mathrm{MPa})$ & 197835 & 197749 \\
\hline
\end{tabular}

Al comparar los resultados de las propiedades de los materiales utilizados para la construcción de las losas (tabla 7), tanto el concreto como el acero utilizado por Carranza (2017) superan la resistencia de los utilizados en este proyecto. Únicamente el módulo de elasticidad del acero fue similar en ambas investigaciones.

Posteriormente se compararon los cortantes experimentales $\left(\mathrm{V}_{\text {experimental }}\right)$ y momentos experimentales $\left(\mathrm{M}_{\text {experimental }}\right.$ ) soportados por los especímenes (ver figura 14). En el caso de las losas reforzadas con el sistema de CFRP, se promediaron los resultados principales de cada investigación. 


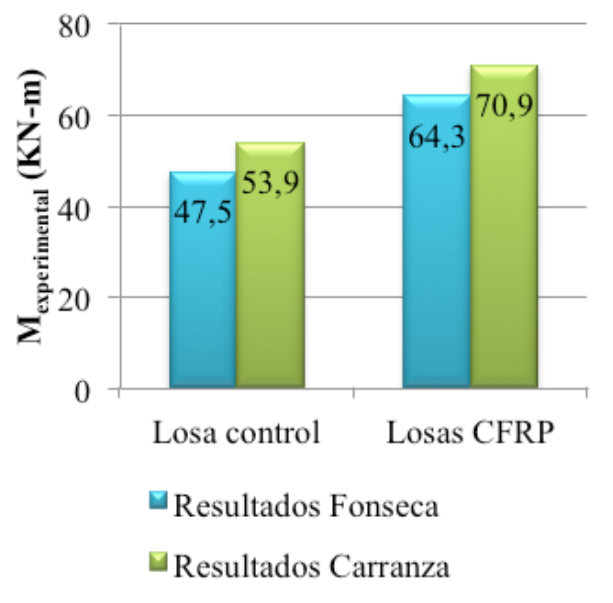

a. Momentos experimentales

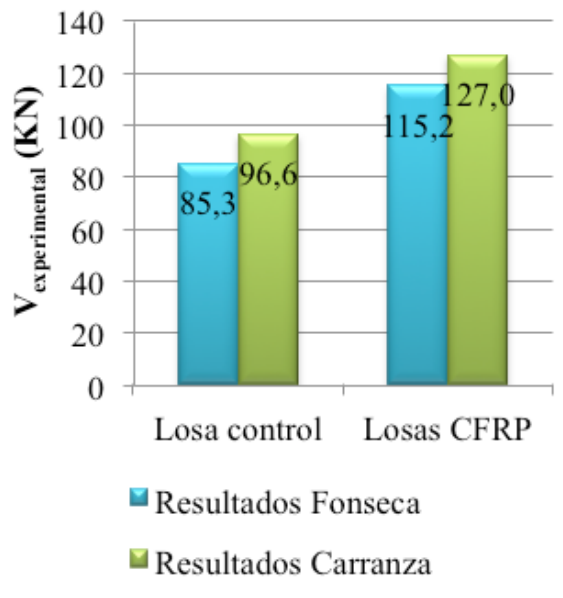

b. Cortantes experimentales

Figura 14. Momentos y cortantes experimentales de ambas investigaciones

De acuerdo con las figuras anteriores, tanto la losa control como las losas reforzadas con CFRP de la investigación de Carranza (2017) tuvieron una mayor capacidad que las losas evaluadas en este proyecto. La resistencia de la losa control de Carranza (2017) fue un $13.9 \%$ superior y la resistencia promedio de las losas reforzadas con CFRP de Carranza (2017) fue un $10.5 \%$ superior.

Se considera que las diferencias en la capacidad de las losas de ambas investigaciones se deben principalmente a las diferencias en las resistencias de los materiales (ver tabla 7); es decir, no se considera que la precarga afectara significativamente la resistencia de los elementos, pues los resultados de ambas investigaciones fueron similares.

A continuación se compara el modo de falla de las losas. En ambas investigaciones las losas control fallaron por una combinación de esfuerzos de flexión y cortante, mientras que las losas reforzadas con CFRP fallaron por grietas de cortante que se extendieron hasta las zonas donde se encontraba la fibra y generaron su desprendimiento. Este comportamiento se debe a que el refuerzo de CFRP aumentó la capacidad de flexión hasta sobrepasar la capacidad en cortante de las losas, por lo que el modo de falla fue en cortante.

Como observación final, en ambas investigaciones se obtuvieron porcentajes de aumento de resistencia similares (tabla 8) lo que puede implicar que el desempeño del refuerzo de CFRP no se ve significativamente afectado por ser utilizado en un elemento reparado. Es decir, el reforzamiento tanto de losas sin daño como losas reparadas, permitió aumentar la capacidad de los elementos en un porcentaje similar. 
Tabla 8. Aumento de resistencia de las losas reforzadas con CFRP con respecto a la losa control

\begin{tabular}{ccc}
\hline & Resultados Fonseca & Resultados Carranza \\
Aumento de resistencia de losas & $36.6 \%$ & $32.6 \%$ \\
CFRP respecto a losa control & & \\
\hline
\end{tabular}

\section{CONCLUSIONES}

- Se construyeron tres especímenes de losas, cuyas características buscaron replicar losas de puentes existentes en Costa Rica. Uno de estos especímenes funcionó como un control y los otros dos fueron reparados y reforzados con el sistema de fibras de carbono.

- Al aplicar un $80 \%$ de la carga última a las losas, se logró generar un estado de agrietamiento que requirió reparación mediante la inyección de epóxico antes de su posterior reforzamiento con el sistema de CFRP.

- El sistema de refuerzo externo con CFRP permitió aumentar la capacidad en flexión de las losas de este proyecto, pues en promedio se presentó un aumento de $36.6 \%$ en las losas reforzadas con CFRP con respecto a la losa control.

- El refuerzo con CFRP aumentó la capacidad en flexión de las losas ensayadas por encima de la capacidad en cortante. Consecuentemente, la falla ocurrió por esfuerzos de cortante que generaron grietas que se extendieron hasta las zonas reforzadas con CFRP y ocasionaron su delaminación.

- La falla de la losa control se dio por una combinación de esfuerzos de cortante y flexión, los cuales fueron visibles en su patrón de agrietamiento.

- Al comparar los resultados, se evidencia que el daño generado por la precarga de las losas no influyó en su capacidad final, pues se obtuvieron resultados cercanos a los obtenidos por Carranza (2017); las diferencias obtenidas entre ambos proyectos se explican principalmente por las diferencias en las resistencias de los materiales utilizados en ambas investigaciones.

- Las losas control de este proyecto y las de Carranza (2017) fallaron en forma similar debido a una combinación de esfuerzos de flexión y cortante.

- Las losas reforzadas con CFRP de este proyecto y las de Carranza (2017) fallaron en forma frágil en cortante; por lo tanto, los diseñadores deben tomar en consideración el tipo de reforzamiento y el aumento de capacidad que se le dará al elemento, con el fin de evitar fallas frágiles.

- El porcentaje de aumento de resistencia de las losas reforzadas con CFRP con respecto a la losa control, en ambos proyectos fue superior al 30\%, lo que demuestra que el sistema de refuerzo externo con CFRP permite obtener incrementos significativos de capacidad. 


\section{REFERENCIAS}

American Standard of Testing and Materials. (2016). ASTM A615 / A615M-16, Standard Specification for Deformed and Plain Carbon-Steel Bars for Concrete Reinforcement. West Conshohocken: ASTM International

Arduini, M., Nanni, A. y Romagnolo, M. (2004). Performance of one-way reinforced concrete slabs with externally bonded fiber-reinforced polymer strengthening. ACI Structural Journal, 101(2), 193-201

Carranza, D. (2017). Evaluación del comportamiento a flexión en losas de puentes reforzadas externamente con fibras de carbono. Proyecto de graduación para optar por el grado de Licenciatura en Ingeniería Civil, Escuela de Ingeniería Civil, Universidad de Costa Rica, San José, Costa Rica.

Mata, J. (2014). Evaluación del comportamiento a flexión en vigas de concreto reforzadas con fibras de carbono, una vez que han sido sometidas a un estado de carga. Proyecto de graduación para optar por el grado de Licenciatura en Ingeniería Civil, Escuela de Ingeniería Civil, Universidad de Costa Rica, San José, Costa Rica.

Osejo, J. (2012). Evaluación del comportamiento de vigas de concreto armado reforzadas externamente con fibras de carbono. Proyecto de graduación para optar por el grado de Licenciatura en Ingeniería Civil, Escuela de Ingeniería Civil, Universidad de Costa Rica, San José, Costa Rica.

Sika Colombia S.A. (2012). Hoja técnica Sikadur-301 Resina de impregnación de alto módulo y alta resistencia. Extraída el 13 de junio, 2017 del sitio web de Sika Costa Rica: http:// cri.sika.com/es/solutions_products/document_download/ht.html?device=desktop\&page=3

Uomoto, T., Mutsuyoshi, H., Katsuki, F. y Misra, S. (2002). Use of fiber reinforced polymer composites as reinforcing material for concrete. Journal of materials in civil engineering, 14(3), 191-209. 
